Publisher: African Networks on Ethnomedicines

Website: //http://journals.sfu.ca/africanem/index.php /AJID

http://dx.doi.org/10.4314/ajid.v6i2.4

\title{
ASSOCIATION OF HIV-INDUCED IMMUNOSUPPRESSION AND CLINICAL MALARIA IN NIGERIAN ADULTS
}

\section{Michael O. Iroezindu ${ }^{1 *}$, Emmanuel I. Agaba ${ }^{2}$, Comfort A. Daniyam ${ }^{2,3}$, Edith N. Okeke ${ }^{2}$, Oche O. Agbaji $^{2,3}$, Patricia A. Agaba ${ }^{3}$, Godwin E. Imade ${ }^{3}$, John A. Idoko ${ }^{2}$}

${ }^{1}$ Department of Medicine, University of Nigeria, Enugu Campus, Nigeria, ${ }^{2}$ Department of Medicine, Jos University Teaching Hospital, Jos, Nigeria, ${ }^{3}$ AIDS Prevention Initiative Nigeria Centre, Jos University Teaching Hospital, Jos, Nigeria

*E-mail: mikezindu@yahoo.com, mikezindu@gmail.com

\begin{abstract}
Despite the growing body of evidence on the interaction between HIV and malaria in sub-Saharan Africa, there is a dearth of data on clinical malaria in HIV-infected patients in Nigeria. We determined the burden of clinical malaria in HIVinfected adult Nigerians and further investigated the association between their immunological status and the rates of clinical malaria. Ninety seven antiretroviral treatment-naïve HIV-infected adults were enrolled in a cross-sectional study from August to December, 2009. The participants had a complete clinical evaluation, thick and thin blood films for malaria parasites and CD4 cell count quantification. Clinical malaria was defined as having fever (temperature $\geq 37.5^{\circ} \mathrm{C}$ or history of fever within 48 hours) and a malaria parasite density above the median value obtained for subjects with co-existing fever and parasitaemia. Clinical malaria was diagnosed in 10 out of 97 patients $(10.3 \%)$. Lower CD4 cell counts were associated with increasing rates of clinical malaria which was $0 \%$ at CD4 cell count of $\geq 500,2.6 \%$ at $200-499$ and $30 \%$ at $<200$ cells $/ \mu \mathrm{L}\left(\chi^{2}=18.3, p=0.0001\right)$. This association remained significant after controlling for other factors in a multivariate analysis ( $\mathrm{AOR}=22.98,95 \%$ C.I: $2.62-20.14, \mathrm{p}$ $=0.005$ ). An inverse relationship between CD4 cell count and parasite density was demonstrated (regression co-efficient $=-$ $0.001, \mathrm{p}=0.0002$ ). More aggressive malaria control measures are highly needed in severely immunosuppressed HIV-infected patients.
\end{abstract}

Key words: HIV, Immunosuppression, Clinical malaria, Adults.

List of non-standard abbreviations

FMOH: Federal ministry of health

JUTH: Jos university teaching hospital

\section{Introduction}

Sub-Saharan Africa with only about $10 \%$ of the world's population is home to $70 \%$ of all the people living with Human immunodeficiency virus (HIV) infection/the acquired immunodeficiency syndrome (AIDS) (UNAIDS, 2010). On the other hand, the WHO (2008) estimates that more than $80 \%$ of the global burden of malaria occurs in sub-Saharan Africa. Therefore, any interaction between the two infections is likely to have significant health consequences in the region especially in countries like Nigeria which currently has the highest malaria burden in sub-Saharan Africa and ranks top among the African nations plagued by HIV/AIDS (FMOH, 2005; WHO, 2008).

The hallmark of HIV infection is progressive immunosuppression affecting both cellular and humoral responses. By far the most important event in the immunopathogenesis of HIV/AIDS is the depletion of CD4 T lymphocytes (Fauci, 1991). In malaria endemic regions, with constant and repeated exposure to malaria infection, natural immunity gradually develops usually from childhood leading to reduced episodes of clinical malaria. The mechanism of malaria immunity requires activation of innate pathways including counter-regulatory cytokines followed by adequate T and B cell responses (Wabwire-Mangen et al., 1989; Troye-Blomberg, 1994). The impact of HIV infection on the immune system has led to researches to unravel the clinical implication of any possible interaction between these two infections in areas where they are endemic.

A number of studies in sub-Saharan Africa have reported increased risk of malaria parasitaemia and clinical malaria in HIV-infected populations compared to HIV negative groups (Patnaik et al., 2005; Whitworth et al., 2000; Francesconi et al., 2001). Among the HIV-infected patients, it has further been demonstrated that malaria parasite density and clinical malaria episodes have an inverse relationship with the immunological status of the patients as reflected by the CD4 cell count (Francesconi et al., 2001; Laufer et al., 2006; French et al., 2001). This emerging evidence is likely to attract additional economic demands to the already overwhelming cost of malaria treatment in the region. Despite the high endemicity of HIV and malaria in Nigeria, there is paucity of data regarding the clinical implication of HIV and malaria interaction in the country. Although 
Publisher: African Networks on Ethnomedicines Website: //http://journals.sfu.ca/africanem/index.php /AJID http://dx.doi.org/10.4314/ajid.v6i2.4 increased prevalence of malaria parasitaemia in HIV-infected populations has been documented in few studies (Akinbo et al., 2009; Uneke et al., 2005; Onyenekwe et al., 2007), clinical malaria was not captured in any of the available reports and nearly all the studies were limited by inability to determine the immunological status of the HIV positive patients. This has created the need for this study which aims to determine the burden of clinical malaria in HIV-infected Nigerians and further investigate the association between the immunological status of HIV-infected patients and the rates of clinical malaria.

\section{Materials and Methods \\ Study setting and design}

The study was carried out at the adult antiretroviral clinic of Jos University Teaching Hospital (JUTH) between August and December, 2009. This tertiary hospital serves as a referral centre to neighbouring states in North-central Nigeria which has the highest burden of HIV/AIDS in the country. The study design was cross sectional.

\section{Case definitions}

Malaria parasitaemia (malaria infection) was defined as a positive malaria smear irrespective of the parasite density (Greenwood, 1997); Fever was defined as documented axillary temperature $\geq 37.5^{\circ} \mathrm{C}$ or a history of fever within the previous 48 hours of the study (Greenwood, 1997; Francesconi, et al., 2001); while Clinical Malaria was defined as having fever (as defined above) and a malaria parasite density above the median value obtained for subjects with co-existing fever and parasitaemia (Francesconi, et al., 2001).

\section{Data collection}

Consecutive ninety seven (97) antiretroviral treatment-naïve HIV-infected patients who were 18 years and above were recruited. An interviewer-administered questionnaire was used to obtain information on the socio-demographic characteristics of the patients and history that may be suggestive of malaria or opportunistic conditions which would serve as exclusion criteria. A detailed physical examination was carried out. The criteria for exclusion from the study were pregnancy, antiretroviral therapy experience, exposure to antimalarial therapy within one month of the study, patients on co-trimoxazole, patients with clinically obvious opportunistic diseases such as tuberculosis, pneumonia, meningitis, sepsis, gastroenteritis and malignancies as well as those on immunosuppressive therapy such as cytotoxics and steroids. HIV infection was confirmed by western blot assay and CD4 T-lymphocyte count was quantified using flow cytometry (Partec, Germany). Thin and thick blood film microscopy were done for each patient using standard protocols for Giemsa staining for identification of malaria parasites and quantification of parasite density respectively (WHO, 2000)Malaria parasite densities were determined using thick films observed for two hundred leucocytes and reported to an average of 8,000 leucocytes $/ \mu \mathrm{L}(\mathrm{WHO}, 2000)$ i.e

Parasite density $($ parasites $/ \mu \mathrm{L}$ of blood $)=\frac{\text { mean asexual parasites counted }}{\text { number of leucocytes (i.e. } 200)} \times 8000$

\section{Ethical considerations}

The study was approved by the Ethics committee of JUTH. Participation was voluntary and informed consent was obtained from the patients. Confidentiality was ensured. Patients with co-existing fever and malaria parasitaemia were treated with antimalarials at no cost to them.

\section{Statistical methods}

Data was analysed using the Epi Info 2005 statistical programme (CDC, Atlanta, Georgia). Means (SD) and proportions were used to describe continuous and categorical variables respectively. The Chi-squared test was used to compare observed differences in proportions while Student t-test was used to assess mean differences. Trend in malaria parasite density was investigated using a linear regression of $\log _{10}$ transformed data. Logistic regression model was used to determine factors independently associated with clinical malaria. A p-value of $<0.05$ was considered significant.

\section{Results \\ Characteristics of the study population}

The characteristics of the study subjects are shown in Table 1. Out of 97 HIV-infected patients recruited, there were 56 females $(57.7 \%)$ and 41 males $(42.3 \%)$. The mean age of the participants was $36 \pm 9$ years. Majority of the participants had some form of formal education (76.3\%), and most of them were employed (76.3\%). Urban dwellers (55.7\%) were more than those 
Publisher: African Networks on Ethnomedicines

Website: //http://journals.sfu.ca/africanem/index.php /AJID

http://dx.doi.org/10.4314/ajid.v6i2.4

residing in rural areas $(44.3 \%)$. The study participants were fairly distributed across the three CD4 cell categories with a small majority (40.2\%) having CD4 cell count of 200-499 cells/L.

Among the study subjects, 38 (39.2\%) had fever, 24 (24.7\%) had malaria parasitaemia while $15(15.5 \%)$ had both fever and malaria parasitaemia irrespective of parasite density otherwise called febrile parasitaemia. The median parasite density among patients with both fever and parasitaemia was 1,400 parasites $/ \mu \mathrm{L}$ and this was adopted as the cut-off parasite density for defining clinical malaria. Out of the 97 patients, $10(10.3 \%)$ fulfilled the clinical malaria case definition.

Table 1. Characteristics of study population

\begin{tabular}{|c|c|}
\hline Characteristic & \\
\hline $\operatorname{Sex}(F / M)$ & $56 / 41$ \\
\hline Age $($ Mean $\pm S D)$, yrs & $36 \pm 9$ \\
\hline Employed (Yes/No) & $74 / 23$ \\
\hline Residence (Urban/Rural) & $54 / 43$ \\
\hline Marital status, n (\%) & \\
\hline Married & $69(71.1)$ \\
\hline Single/Widowed/Separated/Divorced & $28(28.9)$ \\
\hline Educational status, n (\%) & \\
\hline None/Informal & $23(23.7)$ \\
\hline Primary & $28(28.9)$ \\
\hline Secondary & $33(34.0)$ \\
\hline Tertiary & $13(13.4)$ \\
\hline CD4 cell count $(\operatorname{cell} / \mu \mathrm{L}), \mathrm{n}(\%)$ & \\
\hline$\geq 500$ & $28(28.9)$ \\
\hline $200-499$ & $39(40.2)$ \\
\hline$<200$ & $30(30.9)$ \\
\hline Clinical presentation, $\mathrm{n}(\%)$ & \\
\hline Patients with fever irrespective of MP & $38(39.2)$ \\
\hline Patients with MP irrespective of fever & $24(24.7)$ \\
\hline Patients with fever and parasitaemia & $15(15.5)$ \\
\hline Patients with clinical malaria ${ }^{\dagger}$ & $10(10.3 \%)$ \\
\hline
\end{tabular}

$\mathrm{MP}=$ malaria parasitaemia.

${ }^{\dagger}$ Clinical malaria was defined as having fever and malaria parasite density $>1,400$ parasites $/ \mu \mathrm{L}$ which was the median parasite density among patients with co-existing fever and parasitaemia.

\section{Relationship between CD4 cell count and clinical malaria}

The relationship between CD4 cell count and various clinical presentations is shown in Table 2. Lower CD4 cell counts were associated with increasing rates of clinical malaria which was $0 \%$ at CD4 cell count of $\geq 500$ cells $/ \mu \mathrm{L}, 2.6 \%$ at $200-499$ cells $/ \mu \mathrm{L}$ and $30 \%$ at $<200$ cells $/ \mu \mathrm{L}\left(\chi^{2}=18.3, \mathrm{p}=0.0001\right)$. On further analysis, lower CD4 cell counts were also associated with increasing rates of febrile parasitaemia which was $3.6 \%$ at CD4 cell count of $\geq 500$ cells $/ \mu \mathrm{L}, 5.1 \%$ at $200-499$ cells $/ \mu \mathrm{L}$ and $40 \%$ at $<200$ cells $/ \mu \mathrm{L}\left(\chi^{2}=20.0, \mathrm{p}<0.0001\right)$. There was, however, no difference across the CD4 cell categories in terms of having only fever without parasitaemia $\left(\chi^{2}=0.08, \mathrm{p}=0.96\right)$. Those in rural areas were more likely to have clinical malaria than urban dwellers on univariate analysis $\left(18.6 \%\right.$ Vs. $\left.3.7 \%, \chi^{2}=5.7, p=0.02\right)$ but this did not attain statistical significance on multivariate analysis $(\mathrm{AOR}=4.6,95 \%$ C.I: $0.78-27.03, \mathrm{p}=0.09)$. Clinical malaria was not affected by sex $\left(12.2 \%\right.$ in males and $8.9 \%$ in females, $\chi^{2}=$ $0.27, \mathrm{p}=0.60)$ or age $\left(\chi^{2}=0.35, \mathrm{p}=0.99\right)$. The association between low CD4 cell count and clinical malaria remained significant after controlling for other factors in a multivariate analysis ( $\mathrm{AOR}=22.98,95 \%$ C.I: $2.62-20.14, \mathrm{p}=0.005)$ as shown in Table 3 .

\section{Relationship between CD4 cell count and malaria parasite density}

As shown in Figure 1, there was an inverse relationship between CD4 cell count and $\log _{10}$ parasite density (regression co-efficient $=-0.001, \mathrm{p}=0.0002$ ). When the data was analysed categorically, patients with $\mathrm{CD} 4$ cell count $\geq 500$ cells $/ \mu \mathrm{L}$ had a median parasite density of 160 (IQR 80-400) parasites/ $\mu \mathrm{L}$ compared to 400 (IQR 360-600) parasites/ $\mu \mathrm{L}$ for those with CD4 cell count 200-499 cells $/ \mu \mathrm{L}$ and 1,600 (IQR $720-2,200)$ parasites $/ \mu \mathrm{L}$ for CD4 cell count $<200$ cells $/ \mu \mathrm{L}(\mathrm{p}=0.001)$.

\section{Discussion}

Our study showed that $10.3 \%$ of the HIV-infected patients had clinical malaria. This is lower than the rates of $24 \%$ by Patnaik et al. (2005) in Malawi and $22.6 \%$ by Francesconi et al. (2001) in Uganda. This is likely due to differences in the study 
Publisher: African Networks on Ethnomedicines

Website: //http://journals.sfu.ca/africanem/index.php /AJID

http://dx.doi.org/10.4314/ajid.v6i2.4

populations and cut-off parasite densities for clinical malaria case definition. Although the cut off parasite density of $\geq 2000$ parasites $/ \mu \mathrm{L}$ used for clinical malaria case definition in Malawi was higher than that of our study $(>1,400$ parasites $/ \mu \mathrm{L})$, their

Table 2: Clinical presentations of HIV-infected patients according to CD4 cell category

\begin{tabular}{|c|c|c|c|c|}
\hline Characteristic & $\begin{array}{l}\text { Present } \\
\mathrm{n}(\%)\end{array}$ & $\begin{array}{l}\text { Absent } \\
\mathrm{n}(\%)\end{array}$ & $\chi^{2}$ & p-value \\
\hline \multicolumn{5}{|c|}{ CM* (fever and MP density $>1,400$ parasites/ $\mu L)$} \\
\hline$\geq 500 \mathrm{cell} / \mu \mathrm{L}$ & $0(0.0)$ & $28(100)$ & & \\
\hline $200-499$ cell $/ \mu \mathrm{L}$ & $1(2.6)$ & $38(97.4)$ & & \\
\hline$<200$ cell $/ \mu \mathrm{L}$ & $9(30.0)$ & $21(70.0)$ & 18.3 & 0.0001 \\
\hline \multicolumn{5}{|c|}{ Febrile parasitaemia ${ }^{\dagger}$} \\
\hline$\geq 500 \mathrm{cell} / \mu \mathrm{L}$ & $1(3.6)$ & $27(96.4)$ & & \\
\hline $200-499$ cell $/ \mu \mathrm{L}$ & $2(5.1)$ & 37 (94.9) & & \\
\hline$<200$ cell $/ \mu \mathrm{L}$ & $12(40.0)$ & $18(60.0)$ & 20.0 & $<0.0001$ \\
\hline \multicolumn{5}{|c|}{ Fever without malaria parasitaemia } \\
\hline$\geq 500$ cell $/ \mu \mathrm{L}$ & $6(21.4)$ & $22(78.6)$ & & \\
\hline $200-499$ cell $/ \mu \mathrm{L}$ & $8(20.5)$ & $31(79.5)$ & & \\
\hline$<200$ cell $/ \mu \mathrm{L}$ & $7(23.3)$ & $23(76.7)$ & 0.08 & 0.96 \\
\hline
\end{tabular}

${ }^{*} \mathrm{CM}=$ clinical malaria, $\mathrm{MP}=$ malaria parasite ${ }^{\dagger}$ Febrile parasitaemia $=$ co-existing fever and malaria parasitaemia irrespective of parasite density.

Table 3. Factors associated with clinical malaria (multivariate analysis)

\begin{tabular}{llll}
\hline Characteristic & AOR & $\mathbf{9 5 \%} \mathbf{C I}$ & p-value \\
\hline Age & 1.03 & $0.94-1.12$ & 0.52 \\
CD4 cell count $(<200 / \geq 200)$ & 22.98 & $2.62-20.14$ & 0.005 \\
Residence (Rural/Urban) & 4.60 & $0.78-27.03$ & 0.09 \\
Sex (Male/Female) & 1.04 & $0.22-4.81$ & 0.96 \\
\hline
\end{tabular}

$\mathrm{AOR}=$ Adjusted Odds ratio, $\mathrm{CI}=$ confidence interval.

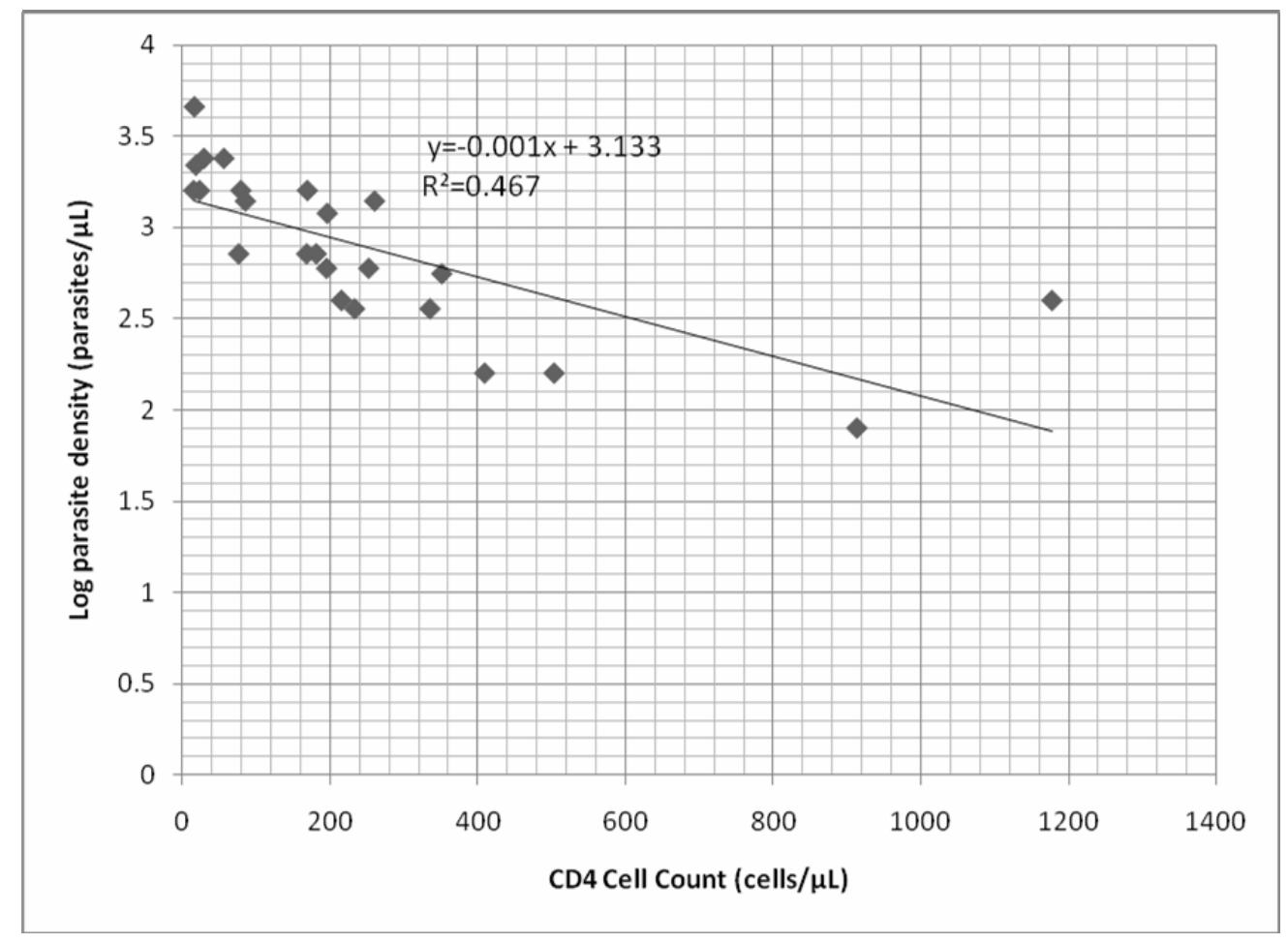

Figure 1: Relationship between CD4 cell count and $\log _{10}$ parasite density 
Publisher: African Networks on Ethnomedicines

Website: //http://journals.sfu.ca/africanem/index.php /AJID

http://dx.doi.org/10.4314/ajid.v6i2.4

study involved an absolutely rural population who are known to have a higher burden of malaria than urban dwellers unlike our study where rural dwellers constituted only $44.3 \%$ of the participants. The sample size in our study was about three times higher than the number of HIV positive patients recruited in Uganda while the cut off parasite density used for clinical malaria case definition in our study was about two and half times higher than theirs. The diverse median parasite densities in these populations reflect the variable burden of malaria transmission and the differences in the antimalarial immunity of these HIV-infected populations at the time of the studies.

It was demonstrated in our study that advancing HIV-induced immunosuppression was associated with increasing rates of clinical malaria of $0 \%$ at CD4 cell count of $\geq 500$ cells $/ \mu \mathrm{L}, 2.6 \%$ at $200-499$ cells $/ \mu \mathrm{L}$ and $30 \%$ at $<200$ cells $/ \mu \mathrm{L}$. Increased risk of clinical malaria at lower CD4 cell counts has similarly been reported in previous studies that investigated the relationship between HIV-induced immunosuppression and clinical malaria in other parts of sub-Saharan Africa (Patnaik et al., 2005; Laufer et al., 2006; French et al., 2001) . Laufer et al. (2006) found that the incidence of clinical malaria was 3-fold higher in HIV positive urban Malawian adults with CD4 cell count of $200-499$ cells $/ \mu \mathrm{L}$ and 4 .4-fold higher in those with CD4 cell count $<200$ cells $/ \mu \mathrm{L}$ compared to individuals with CD4 cell count of $\geq 500$ cells $/ \mu \mathrm{L}$. Patnaik et al. (2005) also reported a hazard ratio of clinical malaria that was two times higher in HIV-infected rural Malawian adults with CD4 cell count $<200$ cells/ $\mu \mathrm{L}$ compared to those with CD4 cell count of 200-399 cells/ $\mu \mathrm{L}$. The incidence of clinical malaria in Uganda was reported to be 140, 93, and 57 cases per 1000 person-years of observation for CD4 cell groups $<200,200-400$ and $>500$ cells $/ \mu \mathrm{L}$ respectively (French et al., 2001).

Higher rates of clinical malaria in these HIV-infected patients residing in malaria endemic regions following advancing immunosuppression can be explained by the impairment in already acquired immune responses to Plasmodium falciparum especially CD4 cell activity and cytokine function leading to defective ability to control parasitaemia which results in higher malaria parasite densities in them (Wabwire-Mangen et al., 1989). In support of this hypothesis, higher malaria parasite densities at lower CD4 cell counts were found in our patients and this has also been reported in other studies (Francesconi et al., 2001; Laufer et al., 2006). Considering the fact that the risk of fever attributable to malaria parasitaemia in an individual increases as the malaria parasite density rises (Greenwood, 1997; Delfini, 1973), these severely immunosuppressed patients with higher parasite densities therefore have a higher risk of clinical malaria.

We also considered the fact that HIV-infected patients with severe immunosuppression are more predisposed to opportunistic infections which are potential confounders for clinical malaria. This was why patients with clinically obvious opportunistic conditions such as tuberculosis, pneumonia, meningitis, gastroenteritis, septicaemia and malignancies were excluded from this study. Moreover, the use of a strict criterion employing a cut-off parasite density for malaria case definition is widely recommended to provide a greater positive predictive value in ascribing a febrile illness to malaria in endemic regions where chance finding of parasitaemia in a febrile patient may occur (Smith et al., 1994; Smith et al., 1995; Schellenberg et al., 1994). Smith et al. (1994) observed that both the sensitivity and specificity of malaria case definitions are maximized at such cutoff parasite density. Our findings were further strengthened by the fact that low CD4 cell count remained significantly associated with higher rates of clinical malaria after adjusting for other factors including place of residence. In order not to completely discountenance the argument that some patients may have fever attributable to malaria parasites at parasite densities below the cut-off, we also determined the relationship between febrile parasitaemia (i.e co-existing fever and parasitaemia irrespective of parasite density) and CD4 cell count which still showed a significant inverse relationship. Further analysis in our study revealed that there was no difference in the rates of fever across the CD4 cell count groups in the absence of parasitaemia which shows that malaria parasites are responsible for the differences observed across the CD4 cell groups in our patients with clinical malaria and febrile parasitaemia.

Socio-demographic characteristics did not have strong associations with clinical malaria in our HIV-infected patients. Though rural dwellers were more likely to have clinical malaria on univariate analysis, this association did not attain statistical significance on multivariate analysis. Neither sex nor age had any significant association with clinical malaria. The tendency of rural dwellers to suffer from clinical malaria compared to urban residents may be attributable to the higher exposure to mosquito bites in rural communities and the observation that rural dwellers in Nigeria do not often engage in malaria control practices (Oladepo et al., 2010). In line with our findings, other studies in Nigeria did not demonstrate any age or sex-dependent differences in the burden of malaria among HIV-infected adults (Uneke et al., 2005; Onyenekwe et al., 2007).

This study was not without limitations. Our inability to quantify antimalarial cytokines such as interferon gamma due to lack of facilities and cost implications made it impossible to assess the impact of HIV infection on the humoral aspect of antimalarial immunity. Exclusion of opportunistic infections on clinical grounds alone could have missed any opportunistic infection that was present but not clinically obvious at the time of the study.

Our study has provided vital local data on clinical malaria in HIV-infected adults and will further improve our understanding of the interaction of HIV and Malaria in Nigeria where both infections are endemic. This revelation justifies the need for aggressive malaria control practices in HIV-infected patients more so in those with severe immunosuppression. Beyond ensuring availability and regular use of insecticide-treated bed nets in HIV positive patients as is applicable in the general population, intermittent presumptive antimalarial chemotherapy as practiced in pregnant women may be considered for HIVinfected patients with severe immunosuppression. 
Publisher: African Networks on Ethnomedicines

Website: //http://journals.sfu.ca/africanem/index.php /AJID

http://dx.doi.org/10.4314/ajid.v6i2.4

\section{Acknowledgement}

We are grateful to the patients for participating in this study. The clinical and laboratory staff of the HIV clinic of JUTH are also appreciated.

\section{References}

1. Akinbo, F. O., Okaka, C. E., Omoregie, R., Mordi, R. and Igbinuwen, O. (2009). Prevalence of malaria and anaemia among HIV-infected patients in Benin City, Nigeria. NZ J. Med. Lab. Sci., 63: 78-80.

2. Delfini, L. F. (1973). The relationship between body temperature and malaria parasitaemia in rural forest areas of Western Nigeria. J. Trop. Med. Hyg., 76: 111-114.

3. Fauci, A. S. (1991). Immunopathogenic mechanisms in human immunodeficiency virus (HIV) infection. Ann. Intern. Med., 114: 678-693.

4. $\quad$ Federal Ministry of Health (2005). Technical Report on National HIV/Syphilis Sero-prevalence Sentinel Survey among Pregnant Women Attending Antenatal Clinics. Department of Public Health National AIDS/STDs Control Program. Abuja, Nigeria.

5. $\quad$ Francesconi, P., Fabiani, M., Dente, M. G., Lukwiya, M., Okwey, R., Ouma, J., Ochakachon, R., Cian, F. and Declich, S. (2001). HIV, malaria parasites, and acute febrile episodes in Ugandan adults: a case-control study. AIDS, 15: 2445-2450.

6. $\quad$ French, N., Nakiyingi, J., Lugada, E., Watera, C., Whitworth, J. A. and Gilks, C. F. (2001). Increasing rates of malaria fever with deteriorating immune status in HIV-1-infected Ugandan adults. AIDS, 15: 899-906.

7. Greenwood, B. M. (1997). The Epidemiology of Malaria. Ann. Trop. Med. Parasit., 91: 763-769.

8. Laufer, M. K., Van Oosterhout, J. J. G., Thesing, P. C., Thumba, F., Zijlstra, E. E., Graham, S. M., Taylor, T. E. and Plowe, C. V. (2006). Impact of HIV-associated immunosuppression on malaria infection and disease in Malawi. J. Infect. Dis., 193: 872-878.

9. Oladepo, O., Tona, G. O., Oshiname, F. O. and Titiloye, M. A. (2010). Malaria knowledge and agricultural practices that promote mosquito breeding in two rural farming communities in Oyo State, Nigeria. Malar. J., 9: 91.

10. Onyenekwe, C. C., Ukibe, N., Meludu, S. C., Ilika, A., Aboh, N., Ofiaeli, N., Ezeani, M. and Onochie, A. (2007). Prevalence of malaria as co-infection in HIV-infected individuals in a malaria endemic area of southeastern Nigeria. $J$. Vector Borne Dis., 44: 250-254.

11. Patnaik, P., Jere, C. S., Miller, W. C., Hoffman, I. F., Wirima, J., Pendame, R., Meshnick, S. R., Taylor, T. E., Molyneux, M. E. and Kublin, J. G. (2005). Effects of HIV-1 serostatus, HIV RNA concentration, and CD4 count on the incidence of malaria infection in a cohort of adults in rural Malawi. J. Infect. Dis., 192: 984-991.

12. Schellenberg, J. R., Smith, T., Alonso, P. L. and Hayes, R. J. (1994). What is clinical malaria? Finding case definition for field research in highly endemic areas. Parasiotol. Today, 10 (11): 439-442.

13. Smith, T., Hurt, N., Teuscher, T. and Tanner, M. (1995). Is fever a good sign for clinical malaria in surveys of endemic communities? Am. J. Trop. Med. Hyg., 52 (4): 306-310.

14. Smith, T., Schellenberg, J. A. and Hayes, R. (1994). Attributable fraction estimates and case definitions for malaria in endemic areas. Stat. Med., 13: 2345-2358.

15. Troye-Blomberg, M. (1994). Human T-cell responses to blood stage antigens in plasmodium falciparum malaria. Immunol. Lett., 41: 103-107.

16. UNAIDS. (2010). UNAIDS report on the global AIDS epidemic. Geneva, Switzerland.

17. Uneke, C. J., Ogbu, O., Inyama, P. U. and Anyanwu, G. I. (2005). Malaria infection in HIV-seropositive and HIVseronegative individuals in Jos, Nigeria. J. Vector Borne Dis., 42: 151-154.

18. Wabwire-Mangen, F., Shiff, C. J and Vlahov, D. (1989). Immunological effects of HIV-1 infection on the humoral response to malaria in an African population. Am. J. Trop. Med. Hyg., 41: 504-511.

19. Whitworth, J., Morgan, D., Quigley, M., Smith, A., Mayanja, B., Eotu, H., Omoding, N., Okongo, M., Malamba, S. and Ojwiya, A. (2000). Effects of HIV-1 and increasing immunosuppression on malaria parasitaemia and clinical episodes in adults in rural Uganda: a cohort study. Lancet, 356: 1051-1056.

20. World Health Organisation. (2008). Roll back malaria partnership: world malaria report. Geneva, Switzerland.

21. World Health Organisation. (2000). Bench aids for the diagnosis of malaria infections. Geneva, Switzerland. 